\title{
Supervivencia y crecimiento de especies con distinta estrategia de vida en plantaciones de áreas quemadas y no quemadas: un estudio de caso con dos especies leñosas en el Chaco Serrano, Argentina
}

\author{
Romina C. Torres ${ }^{1,2, \varpi}$; Melisa Giorgis ${ }^{2,3}$; CeCilia Trillo²; Luis VolkManN²; Pablo \\ Demaio²; JAVIER HeREdia ${ }^{2} \&$ DANiel Renison ${ }^{1,2}$
}

1. Instituto de Investigaciones Biológicas y Tecnológicas, Centro de Ecología y Recursos Naturales Renovables (IIByT - CERNAR - CONICET - Universidad Nacional de Córdoba). 2. ONG Ecosistemas Argentinos. 3 Instituto Multidisciplinario de Biología Vegetal (CONICET - Universidad Nacional de Córdoba). Córdoba, Argentina.

\begin{abstract}
Resumen. Los bosques afectados por fuegos experimentan una creciente demanda de reforestación a fin de reducir la erosión post-fuego y acelerar la recuperación. Bajo la hipótesis de que Acacia caven - una especie dominante en matorrales disturbados - tiene mayor capacidad para tolerar las condiciones post-fuego que Lithraea molleoides (una especie dominante en bosques poco disturbados) evaluamos el desempeño de plantines plantados en áreas quemadas y no quemadas en tres sitios de las Sierras de Córdoba, dos años después de la plantación. La supervivencia varió entre 24 y $92 \%$ según el tratamiento, sin diferencias significativas entre áreas quemadas y no quemadas ni entre especies, pero con diferencias entre sitios de plantación $(\mathrm{P}<0.001)$. El crecimiento también fue muy variable: mientras que en algunos tratamientos, los plantines decrecieron en promedio en altura, en otros crecieron hasta duplicar su altura inicial, sin diferencias significativas entre áreas quemadas y no quemadas. El crecimiento de L. molleoides $(11.4 \pm 1.4 \mathrm{~cm})$ fue mayor que el de $A$. caven $(-2.3 \pm 2.0$ $\mathrm{cm})$ y varió entre sitios de plantación en ambas especies $(\mathrm{P}<0.001)$. Rechazamos la hipótesis planteada ya que ambas especies tuvieron desempeños similares en sitios quemados y no quemados. Nuestra conclusión es que, con fines de restauración de sitios quemados, es recomendable forestar con la especie dominante en bosques poco disturbados ya que la especie dominante en matorrales disturbados se encuentra distribuida ampliamente en la región y su desempeño no fue mejor. Dado que las mayores variaciones se encontraron entre sitios de plantación, destacamos la importancia de estudiar localmente el desempeño de las especies a usar en restauración, en especial en bosques con gran heterogeneidad ambiental como el Chaco Serrano.
\end{abstract}

[Palabras clave: disturbio, especies nativas, reforestación, régimen de fuego]

\begin{abstract}
Aвstract. Outplanting survival and growth of species with different life histories in burned and unburned sites: a case study of two woody species in the Chaco Serrano, Argentina: Post-fire forest restoration is increasingly demanded with the aim of reducing post-fire soil erosion and accelerating forest recovery. Under the hypothesis that Acacia caven, a dominant species in disturbed woodlands, is more tolerant to environmental post-fire conditions than Lithraea molleoides, a dominant species in preserved forests, we assessed the performance of seedlings outplanted to burnt and unburnt areas in three sites of the Córdoba mountains. We registered the survival and growth two years after planting. Survival varied between 24 and $92 \%$ according to treatment, without differences between species and between burnt and unburnt areas but with significant differences among sites $(\mathrm{P}<0.001)$. The growth was variable; in some treatments, seedlings had a mean height lower than plantation height, while in other treatments, seedlings grew up to double its initial height, without differences between burnt and unburnt areas. L. molleoides growth was higher than $A$. caven growth $(11.4 \pm 1.4 \mathrm{~cm}$ and $-2.3 \pm 2.0 \mathrm{~cm}$, respectively) and varied among planting sites $(\mathrm{P}<0.001)$. Contrary to expectations, both species had similar performances in burnt and unburnt areas, while the main differences occurred among sites. To attain restoration goals we recommend planting the dominant species in preserved forest as the dominant species in disturbed woodlands is widely distributed in the region. Our results highlight the importance of studying in each site which species are most adequate to optimize restoration efforts in highly heterogeneous forest as Chaco Serrano.
\end{abstract}

[Keywords: disturbance, native species, reforestation, fire regime]

\section{INTRODUCCIÓN}

En muchas regiones del mundo, el incremento de la frecuencia de fuegos ha provocado cambios profundos en la fisonomía y la composición florística,

\section{Editora asociada: Marina Omacini}

romy.ce2008@gmail.com transformado extensas áreas boscosas en arbustales y pastizales degradados (DíazDelgado et al. 2002; Tálamo \& Caziani 2003; Lloret 2004; De Luis et al. 2006). A la par de estas transformaciones también se producen cambios en las características del suelo, que con

Recibido: 5 de diciembre de 2014, Fin de arbitraje: 16 de enero de 2015, Última versión revisada: 27 de febrero, Aceptado: 8 de abril. 
frecuencia desencadenan procesos de erosión y alteraciones en los regímenes hídricos, con consecuencias económicas graves (Wondzell \& King 2003; Lloret 2004; Bowman et al. 2005). Por ello, la sociedad demanda cada vez más frecuentemente la revegetación asistida de áreas quemadas, ya que a esta práctica se la considera importante en ecosistemas cuya dinámica está condicionada por el fuego (Beyers 2004; Maestre \& Cortina 2004; Pausas et al. 2004; Wang et al. 2006; Siles et al. 2010a).

La restauración activa mediante la plantación de especies leñosas nativas es una de las estrategias que pueden facilitar el proceso de recolonización y sucesión ecológica de áreas incendiadas (Cusack \& Montagnini 2004; Pausas et al. 2004; Rey et al. 2009), en especial en bosques con estación seca, donde el establecimiento a partir de semillas es muy limitado (Torres \& Renison 2012; Torres et al. 2014). La selección de las especies a emplear en la restauración, los requerimientos de hábitat de cada especie, así como las características ambientales del área que se desea restaurar son algunos de los aspectos más importantes a considerar para lograr el éxito de una forestación y alcanzar la comunidad de referencia (Lamb \& Gilmour 2003). Las especies a plantar en áreas quemadas deberían poder soportar una mayor exposición a condiciones ambientales extremas, como insolación elevada, altas temperaturas del suelo y mayor estrés hídrico (Abrahamson 1984; Siles et al. 2010 b).

Las especies dominantes en matorrales disturbados pueden tener un buen desempeño en cuanto a supervivencia y crecimiento en áreas afectadas por fuegos ya que poseen mecanismos para tolerar condiciones estresantes [e.g., condiciones extremas de insolación, bajo contenido de minerales y agua, y temperaturas extremas (Grime 1977; Khurana \& Singh 2001]. Sin embargo, estas especies suelen ser las más abundantes en las áreas quemadas (Gunaratne et al. 2010) y, en consecuencia, el esfuerzo de plantarlas suele ser injustificado. Por otra parte, las especies dominantes en bosques poco disturbados suelen tener una menor tolerancia al estrés y pueden tener dificultades para sobrevivir y crecer en áreas quemadas (Ren et al. 2008; Khurana \& Singh 2001). Las especies dominantes en bosques poco disturbados suelenpreferirsepararealizarenriquecimientos ya que contribuyen a acelerar el proceso de sucesión ecológica y a alcanzar rápidamente la comunidad deseada (Lamb \& Gilmour 2003). Por lo tanto, son fundamentales estudios que ayuden a seleccionar qué especie utilizar para lograr de la forma más eficaz y eficiente el objetivo propuesto.

Por otra parte, en bosques de montaña caracterizados por una gran heterogeneidad topográfica, las condiciones ambientales pueden variar de manera significativa en una escala espacial muy reducida, lo cual determina diferencias en insolación, temperatura y heladas entre distintos sitios. Estas condiciones pueden afectar la supervivencia y el crecimiento de las especies leñosas (Körner 2012). En este sentido, el conocimiento sobre el desempeño de las especies leñosas en áreas quemadas es fundamental no sólo para la recuperación de ecosistemas boscosos, sino también para garantizar la mayor eficiencia en los proyectos de restauración y conservación de estos ecosistemas (Marañón et al. 2004).

El fuego es uno de los principales factores moduladores de la vegetación en las montañas del centro de Argentina (Miglietta 1994; Kunst \& Bravo 2003; Gavier \& Bucher 2004; Giorgis et al. 2011a, 2013). La frecuencia elevada de incendios que existe en la actualidad por las igniciones de origen antrópico (Miglieta 1994; Mari 2006) deviene en una clara necesidad de protección y restauración de las comunidades vegetales. Estudios previos muestran que la regeneración post-fuego de las especies arbóreas ocurre principalmente a partir del rebrote de los individuos ya existentes, mientras que la regeneración de nuevos individuos por semillas es muy escasa (Torres et al. 2014). Estos resultados sugieren que el establecimiento de nuevos individuos se debería promover especialmente por medio de plantaciones. Durante los últimos años se han realizado diversos estudios sobre el efecto del fuego en las comunidades de las sierras de Córdoba (Renison et al. 2002; Verzino et al. 2005; Gurvich et al. 2005; Giorgis et al. 2013; Torres et al. 2014), además de otros estudios tendientes a conocer distintos aspectos de la germinación (Funes et al. 2009; País-Bosch et al. 2012; Torres \& Renison 2012; Valfré-Giorello et al. 2012; Venier 2012) y el establecimiento de las especies nativas (Barchuk et al. 1998; Barchuk \& Díaz 1999, 2000; Verzino et al. 2004; Barchuk et al. 2006a, 2006b; Torres \& Renison 2012; Marcora et al. 2013). Sin embargo, a nuestro entender sólo existe un estudio publicado sobre el desempeño de especies leñosas nativas plantadas en áreas quemadas en las sierras del Centro Argentino, evaluando 
a la especie de altura Polylepis australis (Soteras et al. 2014). En altitudes menores, donde la diversidad de especies arbóreas es mayor (Giorgis et al. 2011a), no se han realizado estudios para evaluar el éxito de plantaciones post-fuego, y sería importante conocer cuáles son las especies más adecuadas para la revegetación de áreas quemadas.

Con el fin de contribuir a evaluar el éxito de la reforestación con especies nativas como estrategia de restauración en áreas incendiadas en las Sierras de Córdoba, estudiamos la supervivencia y el crecimiento de dos especies: una dominante en matorrales disturbados (Acacia caven) y otra dominante en bosques poco disturbados (Lithraea molleoides) (Zak et al. 2004; Giorgis et al. 2011b). Los objetivos fueron: 1) comparar la supervivencia y el crecimiento de plantines de ambas especies plantadas en áreas quemadas y no quemadas en tres sitios, y 2) determinar cuál es la especie más adecuada para revegetar áreas quemadas, en términos de supervivencia y crecimiento. Nuestra hipótesis es que $A$. caven posee más tolerancia a las condiciones ambientales post-fuego de alta insolación y exposición a condiciones ambientales extremas que $L$. molleoides, cuyo establecimiento posiblemente está mediado por procesos de facilitación. En consecuencia, esperamos una mayor supervivencia y crecimiento de $A$. caven en áreas quemadas que en áreas no quemadas y un patrón inverso para L. molleoides.

\section{MATERIALES Y MÉTODOS}

\section{Área de estudio}

Las Sierras de Córdoba, Argentina, están constituidas principalmente por un basamento plutónico metamórfico compuesto por metamorfitas degranogrueso[gneises y migmatitas (Gordillo 1979)]. Las Sierras comprenden cordones longitudinales que se extienden $430 \mathrm{~km}$ de norte a sur y $110 \mathrm{~km}$ de ancho, desde los $29^{\circ} 00^{\prime} \mathrm{S}$ a los $30^{\circ} 30^{\prime} \mathrm{S}$ (Capitanelli 1979; Carignano 1999). Su altitud oscila entre 500 y 2790 m s.n.m. (en el Cerro Champaquí). El clima es sub-húmedo, con tendencia al semi-seco de montaña, y con una estación seca prolongada. Las precipitaciones se concentran entre septiembre y marzo, con un promedio anual de $725 \mathrm{~mm}$. La temperatura media anual es $13.9^{\circ} \mathrm{C}$ (Colladon et al. 2014). El período de ocurrencia de heladas es entre mayo y octubre, con temperatura mínima media mensual de $4.6^{\circ} \mathrm{C}$ (datos tomados con un HOBBO Pro Series Onset, colocado bajo la sombra completa de un arbusto durante los años 2008 a 2010).

La vegetación que cubre las sierras hasta los 1800 m.s.n.m. se encuentra incluida dentro del distrito del Chaco Serrano (Cabido et al. 1998; Giorgis et al. 2011b). Las especies dominantes en bosques poco disturbados son el molle [Lithraea molleoides (Vell.) Engl.] y el orco-quebracho [Schinopsis lorentzii (Griseb.) Engl.], acompañadas por otras especies leñosas, cuya abundancia varía a lo largo de gradientes latitudinales, longitudinales y altitudinales, entre las cuales el espinillo (Acacia caven Molina) es una de las más abundantes en sitios perturbados (Giorgis et al. 2011b). Desde el punto de vista fisonómico, es un bosque heterogéneo producto de la variación espacial en el clima y el uso antrópico, principalmente por fuego, herbivoría e invasión de especies exóticas. En la actualidad, el paisaje está dominado en la zona baja por matorrales secundarios o bosques abiertos y bajos en sitios expuestos, y bosques más densos y altos en quebradas profundas. A mayores altitudes predominan fisonomías de pastizales y arbustales (Luti et al. 1979; Giorgis et al. 2011b).

\section{Especies de estudio}

A Acacia caven (Molina) se la conoce localmente como "espinillo". Es un árbol de hasta $6 \mathrm{~m}$ de altura cuya distribución geográfica abarca Chile, Bolivia, Paraguay, Uruguay, Brasil y Argentina. En nuestro país es dominante en comunidades secundarias en las provincias fitogeográficas del Chaco, Monte, Espinal y en la selva Paranaense (Cabrera 1976). Su presencia es indicadora de alteraciones ambientales (sobrepastoreo y fuego), por lo cual se la considera una especie cicatrizadora (Aronson et al. 1993; Demaio et al. 2002). A. caven es heliófila; rara vez se observan plantines a la sombra de individuos adultos (Gutiérrez \& Armesto 1981; Cabello \& Donoso 2006). En el centro de Argentina es la especie más ampliamente distribuida en las comunidades secundarias de la zona serrana (Giorgis et al. 2011a), que de ahora en más llamaremos matorrales disturbados.

Lithraea molleoides (Vell.) Engl. es conocida localmente como "molle". Es un árbol de hasta 8 $m$ de altura distribuido en Brasil, Bolivia, Uruguay, Paraguay y Argentina. En las Sierras de Córdoba se lo encuentra entre 500 y 1600 m s.n.m. Estudios previos señalan que los renovales son encontrados mayormente a la sombra de arbustos (Martijena 1986; Keegan 1986). Además, en plantaciones tienen un mejor desempeño cuando se encuentran a la sombra de otras plantas leñosas (Verzino et al. 2004; Torres \& Renison 2012), lo que sugiere que es una especie tolerante a la sombra, al menos en estadios tempranos de desarrollo. En la zona serrana del centro de Argentina es una especie frecuente y dominante en comunidades con fisonomía boscosa (Giorgis et al. 2011a), que de ahora en más llamaremos bosques poco disturbados.

\section{Plantaciones}

Para las plantaciones seleccionamos tres sitios que fueron afectados por incendios entre septiembre y 
noviembre de 2004. No contamos con registros de la intensidad de los fuegos, pero sí de su severidad, ya que en los tres sitios el fuego mató la parte aérea de todos los individuos de especies arbóreas en 92\% de las parcelas estudiadas (Torres et al. 2014). Las distancias mínimas y máximas entre sitios fueron 41 y $72 \mathrm{~km}$, respectivamente. Los sitios difieren en su composición florística, en las características ambientales y en su historia de uso.

1) Río Ceballos ( $31^{\circ} 9^{\prime} \mathrm{S} ; 64^{\circ} 8^{\prime} \mathrm{W}$; pendiente: $24 \%$; orientación: sur; altitud: 787 m s.n.m.), ubicado al pie de la ladera este de las Sierras Chicas, cercano a la localidad de Río Ceballos (19133 habitantes) (DGEyC 2008). Este sitio fue afectado por dos fuegos consecutivos en los años 2003 y 2004, y no presentó ganado doméstico ni antes ni después del fuego.

2) Cuesta Blanca ( $31^{\circ} 28^{\prime} \mathrm{S} ; 64^{\circ} 34^{\prime} \mathrm{W}$; pendiente: 17\%; orientación: suroeste; altitud: 750 m s.n.m.), ubicado en la base de la ladera este de las Sierras Grandes y adyacente a la localidad de Cuesta Blanca (371 habitantes) (DGEyC 2008). Este sitio fue afectado por dos fuegos separados por un intervalo de 20 años entre sí (años 1984 y 2004) y presenta ganado en baja densidad (caprino 0.074 UG/ ha y equino $0.015 \mathrm{UG} / \mathrm{ha}$, cargas determinadas por 34 conteos directos desde noviembre de 2004 a febrero de 2006).

3) Uritorco $\left(30^{\circ} 50^{\prime} \mathrm{S}\right.$; $64^{\circ} 29^{\prime} \mathrm{W}$; pendiente: $17 \%$; orientación: este; Altitud: 1000 m s.n.m.), ubicado al pie de la ladera oeste de las Sierras Chicas y a $10 \mathrm{~km}$ de la localidad de Capilla del Monte (10391 habitantes) (DGEyC 2008). Este sitio fue afectado parcialmente por tres fuegos en los años 1984, 1994 y 2004, y en algunos sectores no presenta disturbios en los últimos 60 años. El sitio tenía bajas cargas ganaderas durante el estudio (vacuno $0.026 \mathrm{UG} / \mathrm{ha}$ ) (comunicación personal con propietarios).

Estos sitios fueron usados para estudiar la regeneración espontánea por rebrote y por semilla los cuales se reportaron en Torres et al. (2014). Además, los tres difieren en su composición florística, aun cuando las dos especies seleccionadas para nuestro estudio estaban presentes en todos ellos (Torres et al. 2014).

Entre octubre y noviembre de 2005 plantamos un total de 122 plantines de $A$. caven y 148 plantines de L. molleoides de dos años de edad, adquiridos en viveros locales. Los plantines de $A$. caven se distribuyeron de la siguiente manera: 36 en Río Ceballos, 45 en Cuesta Blanca y 41 en Uritorco. Mientras tanto, los plantines de L. molleoides fueron distribuidos 37 en Río Ceballos, 56 en Cuesta Blanca y 55 en Uritorco. En cada sitio ubicamos al azar 2/3 de los plantines en el área quemada y $1 / 3$ en un área no quemada adyacente. La distancia mínima entre plantines fue $8 \mathrm{~m}$. Al momento de la plantación, los plantines fueron regados por única vez con $10 \mathrm{~L}$ de agua. La altura inicial de los mismos fue $40.4 \pm 1.1$ $\mathrm{cm}$ (A. caven) y $14.8 \pm 0.3 \mathrm{~cm}$ (L. molleoides). Estas diferencias se debieron a que en los viveros no había plantines de tamaño similar para ambas especies. A fin de caracterizar los micrositios de crecimiento, en una parcela circular de $2 \mathrm{~m}$ de radio con centro en cada plantín registramos todas las plantas de especies arbóreas mayores a $30 \mathrm{~cm}$ de altura y les medimos su altura, ancho mayor de la copa y ancho de la copa perpendicular al ancho mayor. Además, estimamos visualmente la proporción de roca y la proporción de vegetación herbácea en cuatro subparcelas de 0.5 × $0.5 \mathrm{~m}$ dentro de la parcela de $2 \mathrm{~m}$ de radio.

En julio y agosto de 2007, después de dos temporadas de crecimiento, registramos la supervivencia y la altura de todos los plantines. Las precipitaciones medias fueron de 525 y $608 \mathrm{~mm}$ para las temporadas de crecimiento 2005/2006 y $2006 / 2007$, respectivamente, mientras que la media para la región es de $623 \mathrm{~mm}$ (media obtenida desde 1992/1993 a 2011-2012) (Colladón 2014). Un nuevo incendio y posterior urbanización afectó las parcelas del área no quemada de Río Ceballos, por lo que este tratamiento fue descartado para el sitio de Río Ceballos.

\section{Análisis estadístico}

Usamos Modelos Lineales Generalizados con asunción de distribución binomial para el análisis de la supervivencia y de distribución normal para el análisis del crecimiento. Dado que no teníamos área control sin quemar, en el sitio Río Ceballos usamos suma de cuadrados tipo I (procedimiento para datos desbalanceados). Consideramos como variables de respuesta a la supervivencia (vivo $=1$; muerto $=0$ ) y el crecimiento (diferencia entre la altura final y la altura inicial) a los dos años de la plantación. Como variables predictoras categóricas incluimos al sitio (con tres niveles: Uritorco, Río Ceballos y Cuesta Blanca), la especie (con dos niveles: A. caven y L. molleoides) y el tratamiento (con dos niveles: área quemada y área no quemada). Además, evaluamos las tres interacciones dobles (especie $x$ tratamiento, especie $\mathrm{x}$ sitio $\mathrm{y}$ tratamiento $\mathrm{x}$ sitio) $\mathrm{y}$ la interacción triple (especie $\mathrm{x}$ tratamiento $\mathrm{x}$ sitio). Además, incluimos como covariables la proporción de roca, la proporción de vegetación herbácea y la biomasa de vegetación leñosa estimada según la fórmula $V=\sqrt{ }\left(4 / 3 . \pi \cdot r^{3}\right)$, donde $r=$ (altura de la planta + ancho mayor de la copa + ancho de la copa perpendicular al ancho mayor)/(3/2). Realizamos las comparaciones entre tratamientos mediante la prueba "a posteriori" de Bonferroni. Se realizaron las pruebas de normalidad y homocedasticidad. Todos los análisis estadísticos se realizaron con el programa Infostat, versión 2013 (Di Rienzo et al. 2013).

\section{REsultados}

\section{Supervivencia}

A dos años de la plantación, la supervivencia de los plantines situados en áreas quemadas 
varió entre 24 y $92 \%$, mientras que en las áreas sin quemar la supervivencia, entre 28 y $79 \%$ (Figura 1a), sin diferencias significativas entre áreas quemadas y sin quemar (MLG, $\mathrm{n}=250$, $\left.\mathrm{gl}=12, \chi^{2}=77.0, P=0.7\right)$. La supervivencia tampoco difirió significativamente entre especies $(P=0.4)$ pero sí varió entre sitios $(P<0.001)$. La interacción sitio $\mathrm{x}$ especie resultó significativa $(P=0.05)$ indicando que la supervivencia de las dos especies siguió un patrón diferente entre localidades. Puntualmente, la supervivencia de $A$. caven fue menor en Cuesta Blanca $(56 \pm 8 \%)$ que en Río Ceballos (85 $\pm 7 \%)$, pero no se

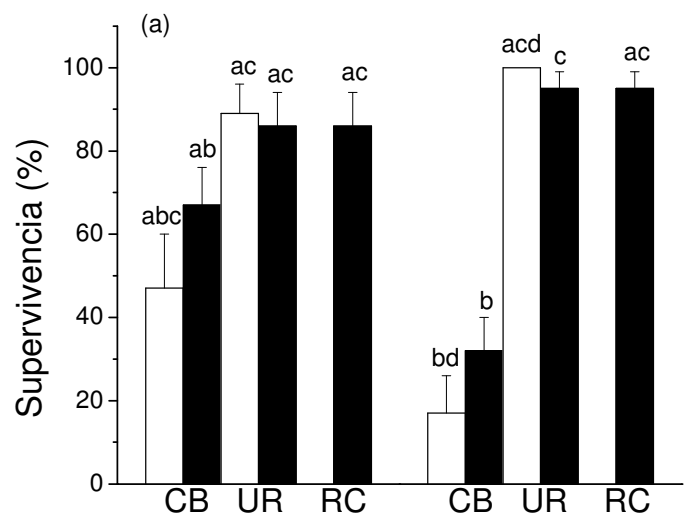

A. caven L. molleoides

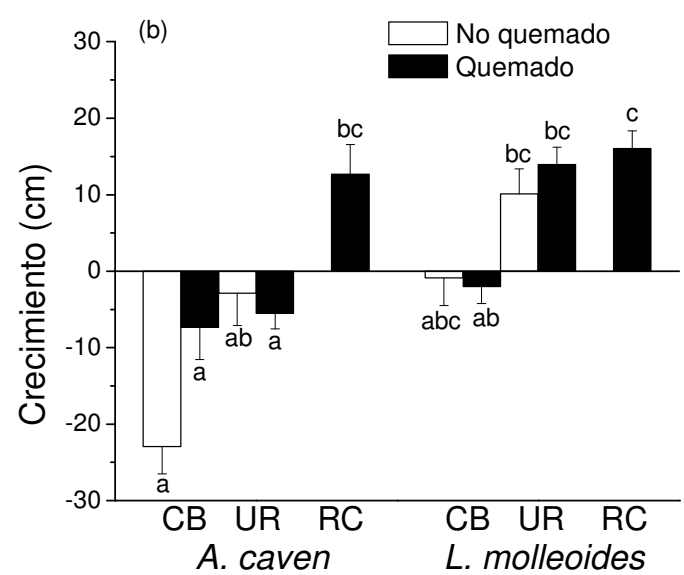

Figura 1. a) Supervivencia y b) crecimiento en altura de plantines de A. caven y L. molleoides al segundo año de plantados en áreas no quemadas y quemadas en tres sitios de las Sierras de Córdoba (media \pm ES). CB: Cuesta Blanca; RC: Río Ceballos; UR: Uritorco. Letras diferentes indican diferencias significativas (prueba a posteriori de Bonferroni).

Figure 1. a) Survival and (b) growth in height of seedlings of $A$. caven and L. molleoides two years after planting in burned and unburned areas at three sites in the Sierras de Córdoba (mean \pm SE). CB: Cuesta Blanca; RC: Rio Ceballos; UR: Uritorco. Different letters indicate significant differences (Bonferroni post-hoc test). encontraron diferencias significativas entre estas localidades y Uritorco $(80 \pm 6 \%)$, mientras que la supervivencia de $L$. molleoides fue mayor en Uritorco $(87 \pm 5 \%)$ y Río Ceballos $(85 \pm 7 \%)$ y menor en Cuesta Blanca (25 $\pm 6 \%$; prueba a posteriori de Bonferroni). No encontramos un efecto significativo de la interacción especie $x$ tratamiento $(P=0.6)$, ni de la interacción sitio $\mathrm{x}$ tratamiento $(P=0.1)$. Tampoco fue significativa la interacción triple especie $x$ tratamiento $\mathrm{x}$ sitio $(P=0.5)$. La supervivencia se relacionó positivamente con la proporción de roca alrededor del plantín $(P=0.04)$, mientras que no fue afectada por la cobertura de vegetación herbácea $(P=0.5)$ ni por la biomasa de vegetación leñosa registrada alrededor del plantín $(P=0.3)$.

\section{Crecimiento}

Dos años después de la plantación, el crecimiento de los plantines no difirió entre áreas quemadas y no quemadas (Figura $1 b$ ) (MLG $\mathrm{n}=165 ; \mathrm{gl}=12 ; \chi^{2}=93.5 ; P=0.6$ ), y fue menor en $A$. caven $(-2.3 \pm 2.0 \mathrm{~cm})$ que en $L$. molleoides $(11.4 \pm 1.4 \mathrm{~cm} ; P<0.001)$. Al igual que lo ocurrido con la supervivencia, el crecimiento de ambas especies difirió significativamente entre sitios $(P<0.001)$ siendo mayor en Río Ceballos (A. caven: $12.7 \pm 3.9 \mathrm{~cm}$; L. molleoides: $16.0 \pm 2.3 \mathrm{~cm}$ ), intermedio en Uritorco (A. caven: $-4.3 \pm 2.2 \mathrm{~cm}$; L. molleoides: $13.0 \pm 1.9 \mathrm{~cm}$ ) y menor en Cuesta Blanca $(A$. caven: $-13.0 \pm 2.9 \mathrm{~cm} ; L$. molleoides: $-1.6 \pm 1.8 \mathrm{~cm}$; Prueba a posteriori de Bonferroni). Además, el crecimiento de cada una de las especies fue diferente entre tratamientos y entre sitios (interacción especie $\mathrm{x}$ tratamiento $\mathrm{x}$ sitio; $P=0.03$ ). En particular, el crecimiento de Acacia caven, tanto en Cuesta blanca como en Uritorco, siempre fue negativo sin diferencias significativas entre área quemada y área no quemada. Por el contrario, el crecimiento de $A$. caven en Río Ceballos fue positivo y significativamente diferente de los otros sitios (Figura 1b). En Cuesta Blanca, por otro lado, el crecimiento de L. molleoides fue negativo y no difirió significativamente entre el área quemada y no quemada, mientras que en Uritorco y en Río Ceballos, el crecimiento de L. molleoides fue positivo y no difirió significativamente entre áreas quemadas y no quemadas (Figura 1b). No detectamos efecto de la interacción especie $x$ tratamiento $(P=0.3)$, ni especie $x$ sitio $(P=0.2)$, ni tratamiento $x$ sitio $(P=0.2)$. El crecimiento de los plantines no se relacionó significativamente con la proporción de roca $(P=0.5)$, la cobertura de vegetación herbácea $(P=0.7)$ ni con el índice de biomasa de la vegetación leñosa circundante $(P=0.6)$. 


\section{Discusión}

Por primera vez reportamos la supervivencia y crecimiento de dos especies en forma comparativa entre sitios quemados y no quemados del Chaco Serrano. Contrariamente a lo esperado, en las especies estudiadas no hubo diferencias en las respuestas entre áreas quemadas y no quemadas, a pesar de tratarse de especies que según la literatura se comportan como dominantes en comunidades que difieren en su grado de perturbación (Aronson et al. 1993; Giorgis et al. 2011a). Ambas especies tuvieron supervivencias y crecimientos muy variables, lo que indica que responden mayormente a condiciones de sitio no evaluadas en este trabajo. Esto pone en evidencia que es necesario desarrollar más estudios orientados a comprender los procesos de reforestación en el Chaco Serrano a fin de mejorar nuestra capacidad de predecir bajo qué situaciones prosperarán las distintas especies arbóreas y por lo tanto, optimizar acciones de restauración de bosques. Los estudios aplicados (como el presente) cumplen el doble objetivo de poner a prueba las nociones ecológicas acerca del ecosistema a restaurar, y al mismo tiempo evaluar estrategias de restauración (Bradshaw 1996).

Las causas por la cuales no encontramos los patrones esperados de un mejor desempeño de la especie dominante en matorrales disturbados en áreas quemadas con respecto a las no quemadas -y lo inverso para la especie dominante en bosques poco disturbados- podrían ser varias. Según nuestros resultados, L. molleoides parece no diferir significativamente de $A$. caven en cuanto a su tolerancia a las condiciones pos fuego, al menos en los primeros años de crecimiento de los plantines. Esto puede deberse a que si bien L. molleoides es una especie dominante en bosques poco perturbados, desde el punto de vista de la estacionalidad de las precipitaciones, el Chaco Serrano es de por sí un ambiente estresante y es posible que muchas de las especies leñosas nativas presenten algún grado de tolerancia al estrés. Estudios previos sobre caracterización del leño de L. molleoides muestran que es una especie adaptada a tolerar estrés hídrico durante la estación de crecimiento (Martijena 1987). Dado que las limitaciones para el establecimiento de una especie leñosa pueden ocurrir en distintas etapas del ciclo de vida, es posible que los patrones predichos se expresen en etapas del ciclo de vida distintas a las evaluadas en este trabajo (e.g., a nivel de depredación de semillas, germinación y establecimiento temprano, o en estadios juveniles más avanzados). Estudios recientes han encontrado que las semillas de A. caven son tolerantes al fuego, lo cual podría explicar que a largo plazo se vuelva dominante en sitios disturbados (Jaureguiberry \& Díaz 2014). Por otra parte, estudios previos en el Chaco Serrano indican que la recuperación del volumen aéreo de la vegetación leñosa pos fuego a través del rebrote es alrededor de 1500 veces más importante que la recuperación por semilla (Torres et al. 2014; Alinari et al. en prensa). Para A. caven y L. molleoides se han reportado valores de supervivencia post-fuego de 89 y $77 \%$, respectivamente, y densidades de plantines de 138 y 724 plantines/ha, respectivamente, sin diferencias significativas entre especies (Torres et al. 2014). Si bien estas especies pueden tener requerimientos de hábitat diferentes, las diferencias en supervivencia pos fuego por rebrote y reclutamiento por semilla son pequeñas, lo cual indica que tienen una capacidad de recuperación similar luego de este tipo de disturbio, y que es posible que no exista una sustitución drástica de especies en respuesta a un solo fuego. Para otras regiones del mundo con fuegos frecuentes también se ha sugerido que las principales especies de plantas de la comunidad están presentes tanto al comienzo de la recuperación pos fuego como en comunidades donde el fuego ha sido excluido por varias décadas debido a altas supervivencias por rebrote (Capitanio \& Carcaillet 2008). Descartaríamos a la falta de potencia estadística como una de las causas de no haber detectado diferencias post-fuego en el presente estudio ya que las diferencias entre sitios se pudieron detectar de forma adecuada con los tamaños muestrales usados.

Con respecto al crecimiento, destacamos el bajo crecimiento de $A$. caven en comparación con L. molleoides, a pesar de considerarse a la primera como una especie cicatrizadora del Bosque Chaqueño Serrano (Giorgis et al. 2011b) y de varios ecosistemas de Sudamérica (Aronson et al. 1993). Esto puede deberse a que una menor tasa de crecimiento es una estrategia más conservadora que le permite a las especies como A. caven tolerar diversas situaciones de estrés. En cambio, la tasa de crecimiento más elevada de L. molleoides sería producto de una capacidad mayor para captar recursos, lo que le permitiría convertirse en dominante en ambientes con una alta competencia, como los ambientes poco disturbados (Grime 2006). Por otra parte, en el momento de la plantación, los plantines de $A$. caven eran más grandes 
que los plantines de L. molleoides, por lo cual pudieron sufrir un mayor estrés hídrico por la pérdida de raíces durante el trasplante. En el futuro, estos aspectos relacionados con el tamaño y edad de los plantines empleados en la reforestación así como la relación biomasa aérea/raíz al momento de ser plantados necesitan ser explorados para mejorar nuestro conocimiento acerca de cómo restaurar áreas quemadas del Chaco Serrano.

Las principales diferenciasenlasupervivencia y el crecimiento de las especies aquí estudiadas se encontraron entre sitios, lo que indica que las variaciones climáticas y edáficas entre sitios pueden ser más importantes para el éxito de la forestación que las características de las especies empleadas y los factores asociados al fuego. Estudios previos han mostrado que la recuperación de la vegetación después del fuego puede variar entre sitios, en especial en bosques con una gran heterogeneidad ambiental [como es el caso del Chaco Serrano (Torres et al. 2014)], pero también en otro tipo de bosques secos para los cuales se ha recomendado tener en cuenta el sitio de plantación para una optimización de los resultados (Âlvarez-Aquino et al. 2004). En el sitio Cuesta Blanca, donde los plantines tuvieron menor supervivencia y crecimiento, estudios previos demuestran que la recuperación de la vegetación después del fuego por rebrote también fue menor que en los otros dos sitios (Torres et al. 2014). Esta menor capacidad de recuperación de la vegetación post-fuego posiblemente se deba a diferencias regionales en la temperatura, precipitaciones o tipos de suelo, ya que la historia de fuegos de Cuesta Blanca es similar a la de los otros sitios. Además, Cuesta Blanca presentó ganado caprino a bajas densidades (0.074 cabras/ha), lo que puede incidir negativamente en el establecimiento de los plantines de forma directa a través del ramoneo, y de forma indirecta al retrasar la recuperación de la vegetación e incrementar la erosión del suelo (Johansson et al. 2009). Esto ha sido reportado en nuestra región para otras especies (Torres et al. 2008; Marcora et al. 2013; Renison et al. 2015). En cambio, la localidad de Río Ceballos se ubica en un sitio con mayor humedad según un gradiente de precipitaciones que disminuye de este a oeste, mientras que la localidad de Uritorco (donde también se registró una mayor supervivencia y crecimiento que en Cuesta Blanca) presenta un mejor estado de conservación del bosque y ganado a cargas muy bajas.

\section{IMPLICANCIAS PARA LA RESTAURACIÓN}

Sugerimos que con fines de restauración de áreas quemadas convendría concentrar los esfuerzos en plantar la especie L. molleoides, dominante en bosques poco perturbados, ya que $A$. caven tuvo una supervivencia similar y un menor crecimiento, en las sierras se encuentra en mayor abundancia y —además - tiene buena capacidad de rebrote pos fuego (Giorgis et al. 2011b; Torres et al. 2014). También destacamos la importancia de estudiar localmente la supervivencia y crecimiento de las especies a fin de desarrollar estrategias de restauración exitosas. Por último, nuestros resultados muestran las dificultades que se presentan al tratar de aplicar los conocimientos existentes hasta el momento acerca de la autoecología de las especies en la práctica de la restauración, y sugieren que aún faltan muchos estudios sobre la autoecología de las especies arbóreas nativas con el fin de poder realizar predicciones más acertadas sobre los sitios de plantación más adecuados.

\section{BiBLIOGRAFÍA}

AbraHAMSON, WG. 1984. Post-fire recovery of Florida lake wales ridge vegetation. American Journal of Botany, 71: 9-21.

Alinari, J; A von Müller \& D Renison. En prensa. The contribution of fire damage to restricting high mountain Polylepis australis forests to ravines: Insights from an unreplicated comparison. Ecol. Aust.

Álvarez-Aquino, C; G Williams-Linera \& AC Newton. 2004. Experimental native tree seedling establishment for the restoration of a Mexican cloud forest. Restor. Ecol., 12:412-418.

Aronson, J; C Floret; E Le Floc' H; C Ovalle \& R Pontanier. 1993. Restoration and rehabilitation of degraded ecosystems in arid and semi-arid lands. II. Case studies in southern Tunisia, central Chile and northern Cameroon. Restor. Ecol., 1:168-187.

BarchuK, AH; EB Campos; C Oviedo \& MP Díaz. 2006a. Supervivencia y crecimiento de plántulas de especies leñosas del Chaco Árido sometidas a remoción de la biomasa aérea. Ecol. Aust., 16:47-61.

BARChUK, AH; MP Díaz; F CASANOVES; MG BALZARINI \& UO KARLIN. 1998. Experimental study on survival rates in two arboreal species from the Argentinian Dry Chaco. For. Ecol. and Manag., 103:203-210.

BARChUK, AH; MR IgLESIAS \& C OvIEDO. 2006b. Rebrote basal de Aspidosperma quebracho blanco en estado de plántula: mecanismo de persistencia en el Chaco Árido. Ecol. Aust., 16:197-205.

BARCHUK, AH \& MP DíAz. 1999. Regeneration and structure of Aspidosperma quebracho-blanco Schl. in the Arid Chaco (Córdoba, Argentina). For. Ecol. and Manag., 118:31-36.

BarchuK, AH \& MP Díaz. 2000. Vigor de crecimiento y supervivencia de plantaciones de Aspidosperma quebracho-blanco y de Prosopis chilensis en el Chaco árido. Quebracho, 8:17-29. 
Beyers, JL. 2004. Post-fire seeding for erosion control: effectiveness and impacts onnative plant communities. Conserv. Biol., 18:947-956.

Bowman, DMJS; JK Balch; P Artaxo; WJ Bond; JM Carlson; ET AL. 2005. Fire in the Earth System. Science, 324:481-484.

BRADSHAW, AD. 1996. Underlying principles of restoration. Can. J. Fish. Aquat. Sci., 53:3-9.

Cabello, A \& C Donoso. 2006. Acacia caven (Mol.) Mol. Pp. 126-134 en: Donoso Zegers, C (ed.). Las especies arbóreas de los bosques templados de Chile y Argentina. Autoecología. Marisa Cuneo Ediciones. Valdivia, Chile.

Cabido, M; G Funes; E Pucheta; F Vendranimi \& S Díaz. 1998. A Chorological analysis of the mountains from Central Argentina. Is all what we call Sierra Chaco really Chaco? Contribution to the study of the flora and vegetation of the Chaco. XII. Candollea, 53:321-331.

CABrera, A. 1976. Regiones fitogeográficas argentinas. Segunda ed. Enciclop. Arg. Agric. y Jardinería. ACME, Buenos Aires.

Capitanelli, J. 1979. Clima. Pp. 45-138 en: Vázquez, J; R Miatello \& M Roque (eds.). Geografía Física de la Provincia de Córdoba. Buenos Aires.

Capitanio, R \& C CARCAILlEt. 2008. Post-fire Mediterranean vegetation dynamics and diversity: A discussion of succession models. For. Ecol. and Manag., 255:431-439.

Carignano, CA. 1999. Late Pleistocene to recent climate change in Córdoba Province, Argentina: Geomorphological evidence. Quaternary International, 57/58:117-134.

Colladón, L. 2014. Anuario pluviométrico 1992/93-2011/ 12. Cuenca del Río San Antonio. Sistema del Río Suquía - Provincia de Córdoba. Instituto Nacional del Agua (INA). Centro de la Región Semiárida (CIRSA), Córdoba, Argentina.

CusaCk, D \& F MONTAGNInI. 2004. The role of native species plantations in recovery of understory woody diversity in degraded pasturelands of Costa Rica. For. Ecol. and Manag., 188:1-15.

De Luis, M; J Raventós \& JC González-Hidalgo. 2006. Post-fire vegetation sucession in Mediterranean gorse shrublands. Acta Oecol., 30:54-61.

Demaio, P; UO Karlin \& M Medina. 2002. Árboles nativos del centro de Argentina. Editorial L.O.L.A.

DGEyC. 2008. Censo Provincial de Población 2008. Dirección general de estadísticas y censos. Gobierno de la provincia de Córdoba. Consultado en: estadistica.cba.gov.ar/ Poblacion/Censo2008/tabid/462/language/es-AR/ Default.aspx

Di Rienzo, JA; F CASANOVES; MG BAlZARINI; L GonZÁlez; ET AL. 2013. InfoStat, versión 2013, Grupo InfoStat, FCA, Universidad Nacional de Córdoba, Argentina.

Díaz-Delgado, R; F lloret; X Pons \& J Terradas. 2002. Satellite evidence of decreasing resilience in Mediterranean plant communities after recurrent wildfires. Ecology, 8:2293-2303.

Funes, G; S DíAz \& P VENIER. 2009. La temperatura como principal determinante de la germinación en especies del Chaco seco de Argentina. Ecol. Aust., 19:129-138.

GAVIER, GI \& EH Bucher. 2004. Deforestación de las Sierras Chicas de Córdoba (Argentina) en el período 1970-1997. Academia Nacional de Ciencias, Córdoba, Argentina. Miscelánea, 101.

Giorgis, MA; M Cabido \& AM Cingolani. 2011a. Caracterización orística y estructural del Bosque Chaqueño Serrano. Córdoba Argentina. Editorial Académica
Española, Saarbrücken.

Giorgis, MA; AM Cingolani; F. Chiarini; J Chiapela; G BARBOZA; ET AL. 2011b. Composición florística del Bosque Chaqueño Serrano de la Provincia de Córdoba, Argentina. Kurtziana, 36:9-46.

Giorgis, MA; M CABido \& AM Cingolani. 2013. El efecto del fuego y las características topográficas sobre la vegetación y las propiedades del suelo en la zona de transición entre bosques y pastizales de las sierras de Córdoba, Argentina. Boletín de la Sociedad Argentina de Botánica, 48:493-513.

GoRDILLO, CE. 1979. Observaciones sobre la petrología de las rocas cordieríticas de las Sierras de Córdoba. Boletín Academia Nacional de Ciencias, 53:3-44.

GRIME, JP. 1977. Evidence for the existence of three primary strategies in plants and its relevance to ecological and evolutionary theory. The American Naturalist, 111:11691194.

GRIME, JP. 2006. Plant strategies, vegetation processes, and ecosystem properties. John Wiley \& Sons.

GUNARATNE, AMTA; CVS GUNATILLEKE; IAUN GUNATILLEKE; HMSP Madawala WeERASINGHEN \& DFRP BuRsLem. 2010. Barriers to tree seedling emergence on human-induced grasslands in Sri Lanka. J. Appl. Ecol., 47:157-165.

Gurvich, DE; L EnRICo \& AM Cingolani. 2005. Linking plant functional traits with post-fire sprouting vigour in woody species in central Argentina. Aust. Ecol., 30: 789-796.

Gutiérrez, J \& JJ Armesto. 1981. El rol del ganado en la dispersión de Acacia caven (Leguminosae). Santiago, Chile. Ciencia e Investigación Agraria, 8:3-8.

JAUREGUIBERRY, P \& S Díaz. 2014. Post-burning regeneration of the Chaco seasonally dry forest: germination response of dominant species to experimental heat shock. Oecología, 177:689-699.

Johansson, M; T RoOKE; M Fetene \& A GRANSTRÖM. 2009. Browser selectivity alters post-fire competition between Erica arborea and E. trimera in the sub-alpine heathlands of Ethiopia. Plant Ecol., 207:149-160.

KeEgan, JM. 1986. Dinámica poblacional de "coco" y "molle". Pp. 462 en: Luti, R (coord.). MAB 6 Efecto de las actividades humanas sobre los ecosistemas montañosos $y$ de tundra. Programa de investigación integrada y entrenamiento en la región andina y montañas extraandinas. Unesco/MAB-PNUMA-CERNAR. Proyecto regional andino: Pachón-Achala.

KhuRAna, AE \& JS Singh. 2001. Ecology of tree seed and seedlings: Implications for tropical forest conservation and restoration. Current Science, 80:748-757.

KÖRNER, C. 2012. Alpine treelines: Functional ecology of the global high elevation tree limits. Springer, Basel.

KunST, C \& S Bravo. 2003. Ecología y régimen de fuego en la región chaqueña argentina. Pp. 331 en: Kunst, C; S Bravo \& JL Panigatti (ed.). Fuego en los ecosistemas argentinos.

LAMB, D \& D GILMOUR. 2003. Rehabilitation and restoration of degraded Forests. IUCN, Gland, Switzerland and Cambridge, UK and WWF, Gland, Switzerland. Pp. 110.

LLORET, F. 2004. Régimen de incendios y regeneración. Pp. 101-126 en: Valladares, F (ed.). Ecología del bosque mediterráneo en un mundo cambiante. Ministerio de Medio Ambiente. EGRAF, S.A., Madrid.

Luti, R; MA Bertrán de Solís; FM Galera; N Müller de FERREIRA; M BERZAL; ET AL. 1979. Vegetación. Pp. 297-368 en: Vázquez, J; R Miatello \& M Roque. Geografía Física 
de la Provincia de Córdoba. Buenos Aires.

Maestre, FT \& J Cortina. 2004. Are Pinus halepensis plantations useful as a restoration tool in semiarid Mediterranean areas? For. Ecol. and Manag., 198:303317.

Marañón, T; JJ Camarero; J Castro; M Díaz; JM Espelta; ET AL. 2004. Heterogeneidad ambiental y nicho de regeneración. Pp. 69-99 en: Valladares, F. Ecología del bosque mediterráneo en un mundo cambiante. Ministerio de Medio Ambiente, EGRAF, S.A., Madrid.

Marcora, PI; D Renison; AI País-Bosch; MR Cabido \& PA TECCO. 2013. The effect of altitude and grazing on seedling establishment of woody species in central Argentina. For. Ecol. and Manag., 291:300-307.

MARI, NA. 2006. Estudio de la ocurrencia de incendios en el Valle de Punilla, Córdoba. Tesis de grado Licenciatura en Gerenciamiento Ambiental. Universidad de Ciencias Empresariales y Sociales. Córdoba, Argentina.

MartijenA, N. 1986. Estructura y estado poblacional del molle en Vaquerías. Pp. 462 en: Luti, R (coord.). MAB 6 Efecto de las actividades humanas sobre los ecosistemas montañosos y de tundra. Programa de investigación integrada y entrenamiento en la región andina y montañas extra-andinas. Unesco/MAB-PNUMACERNAR. Proyecto regional andino: Pachón-Achala.

MARTIJENA, N. 1987. Wood anatomy of Lithraea ternifolia (Gill.) Barkley \& Rom. (Anacardiaceae). IAWA Bulletin, 8:47-52.

Miglietta, S. 1994. Patrón de ocurrencia de incendios y su efecto sobre la vegetación en el Bosque Serrano de Córdoba. Tesis de maestría. Universidad Nacional de Córdoba, Córdoba.

Pais-Bosch, AI; PA Tecco; G Funes \& M Cabido. 2012. Efectos de la temperatura en la regeneración de especies leñosas del Chaco Serrano e implicancias en la distribución actual y potencial de bosques. Boletín de la Sociedad Argentina de Botánica, 47:401-410.

Pausas, JG; C Bladé; A Valdecantos; JP Seva; D Fuentes; ET AL. 2004. Pines and oaks in the restoration of Mediterranean landscapes of Spain: New perspectives for an old practice - a review. Plant Ecol., 171:209-220.

ReN, H; L YANG \& N LiU. 2008. Nurse plant theory and its application in ecological restoration in lower subtropics of China. Progress in Natural Science, 18:137-142.

Renison, D; AM Cingolani \& R SuÁRez. 2002. Efectos del fuego sobre un bosquecillo de Polylepis australis (Rosaceae) en las montañas de Córdoba, Argentina. Rev. Chil. Hist. Nat., 75:719-727.

Renison, D; Mp Chartier; M Menghi; Pi Marcora; Rc TORRES; ET AL. 2015. Spatial variation in tree demography associated to domestic herbivores and topography: Insights from a seeding and planting experiment. For. Ecol. and Manag., 335:139-146.

Rey, PJ; G Siles \& JM Alcántara. 2009. Communitylevel restoration profiles in Mediterranean vegetation: nurse-based vs. traditional reforestation. J. Appl. Ecol., 46:937-945.
Siles, G; PJ Rey \& JM Alcántara. 2010a. Post-fire restoration of Mediterranean forests: Testing assembly rules mediated by facilitation. Basic Appl. Ecol., 11: 422-431.

Siles, G; PJ Rey; JM Alcántara; JM Bastida \& JL Herreros. 2010b. Effects of soil enrichment, watering and seedling age on establishment of Mediterranean woody species. Acta Oecol., 36:357-364.

SOTERAS, F; D RENISON \& AG BECERRA. 2014. Restoration of high altitude forests in an area affected by a wildfire: Polylepis australis Bitt. seedlings performance after soil inoculation. Trees, 28:173-182.

TÁlamo, A \& M Caziani. 2003. Variation in woody vegetation among sites with different disturbance histories in the Argentine Chaco. For. Ecol. and Manag., 184:79-92.

Torres, RC; D Renison; I Hensen; R SuÁrez \& L Enrico. 2008. Polylepis australis' regeneration niche in relation to seed dispersal, site characteristics and livestock density. For. Ecol. and Manag., 254:255-260.

Torres, RC \& D Renison. 2012. Regeneración y respuesta a disturbios en el Bosque Chaqueño Serrano. Recuperación natural y asistida de tres especies arbóreas (Córdoba, Argentina). Editorial Académica Española, Saarbrücken.

Torres, RC; MA Giorgis; C Trillo; L VolkMANn; P Demaio; ET AL. 2014. Post-fire recovery occurs overwhelmingly by resprouting in the Chaco Serrano forest of Central Argentina. Aust. Ecol., 39:346-354.

Valfré-Giorello, TA; L Ashworth \& D Renison. 2012. Patrones de germinación de semillas de Sebastiania commersoniana (Baillon) Smith \& Downs (Euphorbiaceae), árbol nativo del Chaco Serrano de interés en restauración. Ecol. Aust., 22:92-100.

VENIER, P. 2012. El rol de los caracteres regenerativos en la coexistencia de plantas. Un estudio sobre cinco especies de Acacia en los bosques semiáridos de Córdoba, Argentina. Editorial Académica Española, Saarbrücken. Pp. 160.

Verzino, G; J Joseau; M Dorado; E Gellert; S Rodríguez REARTES; ET AL. 2005. Impacto de los incendios sobre la diversidad vegetal, Sierras de Córdoba, Argentina. Ecol. Apl., 4:25-34.

Verzino, G; J Joseau; MP díaz \& M Dorado. 2004. Comportamiento inicial de especies nativas del Chaco Occidental en plantaciones en zonas de pastizales de altura de las Sierras de Córdoba, Argentina. Bosque, 25:53-67.

WANG, X; HS HE; X Li; Y ChanG; Y Hu; ET AL. 2006. Simulating the effects of reforestation on a large catastrophic fire burned landscape in Northeastern China. For. Ecol. and Manag., 225:82-93

Wondzell, SM \& JG KING. 2003. Postfire erosional processes in the Pacific Northwest and Rocky Mountain regions. For. Ecol. and Manag., 178:75-87.

ZAK, MR; M CABIDO \& JG Hodgson. 2004. Do subtropical seasonal forests in the Gran Chaco, Argentina, have a future? Biol. Conserv., 120:589-598. 